\title{
(6)
}

Mercedes Alda García*

Ana García Taboada*

Isabel Marco Sanjuán*

\section{APARICIÓN Y CRECIMIENTO DE LOS ETF EN ESPAÑA Una interesante inversión alternativa poco conocida en tiempos de crisis}

A pesar de la reciente aparición de los fondos cotizados (Exchange Traded Funds, ETF) en España, estos han alcanzado una importancia considerable, con una inversión superior a los 44.560 millones de euros en junio de 2015. Los ETF son productos híbridos entre los fondos de inversión y los títulos de renta variable, que replican un índice y cotizan en mercados regulados. Se han convertido en una inversión estratégica en periodos de crisis dada su gran accesibilidad, por lo que analizamos sus características, principales magnitudes y evolución, así como las ventajas respecto a los fondos tradicionales de este prometedor producto de inversión.

Palabras clave: ETF, inversión colectiva, fondos cotizados, producto de inversión.

Clasificación JEL: D53, G23.

\section{Introducción}

A lo largo de las últimas décadas la inversión en productos de inversión colectiva ha experimentado un crecimiento exponencial en España. Entre estos productos se encuentran los Fondos de Inversión Cotizados, conocidos también por sus siglas en inglés: ETF, Exchange Traded Funds, que pese a su reciente creación en España, en 2006, han presentado un crecimiento notable, alcanzando una inversión superior a los 44.560 millones de euros en junio de $2015^{1}$.

Los ETF son instrumentos de inversión híbridos entre los fondos de inversión y las acciones, de manera que las participaciones del fondo son negociadas en Bolsas de Valores, siendo su objetivo principal la

* Universidad de Zaragoza.

Versión de septiembre de 2015

1 Bolsas y Mercados Españoles BME (www.bolsasymercados.es). réplica de un índice financiero. Esta gestión indexada o pasiva, réplica de un índice, conlleva la compra de todos los valores que integran el índice en la misma proporción.

Los fondos cotizados, poco conocidos entre los inversores particulares en España, ofrecen interesantes características y opciones de inversión, especialmente ante situaciones coyunturales de bajos tipos de interés, como la actual. Por ello analizamos este producto alternativo de inversión que ofrece atractivas posibilidades, bajo un marco de regulación legal y de protección del inversor.

A pesar de la creciente importancia de los ETF en el mercado español, y dado lo que nos es conocido, no existen estudios previos que analicen su evolución y características, por lo que en este trabajo se realiza un estudio pormenorizado con el objeto de conocer mejor la situación de este producto de inversión menos tradicional. 
El artículo se estructura en los epígrafes que desarrollamos a continuación. En el apartado dos se realiza la descripción del producto, su tipología y normativa reguladora, analizando sus principales características y atractivas diferencias con respecto a los fondos de inversión tradicionales. En el apartado tres se muestra el funcionamiento y método para replicar índices. En el cuarto epígrafe se analizan las principales magnitudes de este segmento del mercado de inversión y su importancia en el mercado de capitales. Este apartado se completa con el análisis detallado de los ETF indexados a índices de la familia IBEX, dado que son los más representativos en cuanto a volumen de negociación en el mercado español. En el último apartado se recogen las principales conclusiones.

\section{Los ETF: origen y características}

\subsection{Origen}

El origen de los ETF se encuentra vinculado a los fondos SPDR (Standard \& Poor's Depositary Receipts), que empezaron a comercializarse durante los años noventa en Estados Unidos, con los que se buscaba replicar índices bursátiles. No obstante, tal y como indica Gastineau (2010), el primer fondo cotizado aparece en Canadá en la Bolsa de Toronto en 1989, y el primer fondo cotizado en Estados Unidos no aparece hasta 1993, lanzado por State Street Global Advisors, bajo la denominación The SPDR fund. Este fondo seguía al índice Standard \& Poor's 500 y, actualmente, con el nombre SPDR S \& P 500 es el ETF más conocido del mercado americano. Desde entonces, el desarrollo de los ETF ha sido constante, experimentando un crecimiento del 30 por 100 en los últimos diez años, y alcanzando una inversión de 2,6 billones de dólares a nivel mundial durante el tercer trimestre de 2014 (Blackrock, 2014; Hurlin et al. 2015).

La llegada a Europa de estos productos se produce en el año 2000, pero no es hasta 2006 cuando comienzan a comercializarse en España, tras la aprobación del Reglamento de Instituciones de Inversión Colectiva (Real Decreto 1309/2005, de 4 de noviembre). Legalmente, y a todos los efectos, en España, los ETF son fondos de inversión que siguen un estilo de gestión pasiva indexada, cuya principal diferencia con un fondo tradicional es que las participaciones se negocian y liquidan en el mercado bursátil como si de acciones se tratase.

En sus inicios los ETF eran negociados en su mayoría por inversores institucionales, principalmente realizando operaciones de mantenimiento de efectivo durante cambios de estrategia de inversión; sin embargo, en la actualidad también son contratados por inversores minoristas, gracias a las mejoras experimentadas en su operativa y accesibilidad, así como a la amplia expansión que han vivido en los mercados internacionales.

\subsection{Características de los ETF en España y diferencias con los fondos de inversión no cotizados}

Los fondos cotizados comparten muchas de sus características con los fondos de inversión tradicionales, pero presentan una gran diferencia en cuanto a su política de inversión, que consiste, en principio, en replicar a un índice de referencia.

El patrimonio del fondo se divide en un conjunto de participaciones que al ser adquiridas o suscritas por los inversores les confieren la condición de partícipes. La unidad mínima de contratación es una participación, lo cual facilita el acceso al mercado a todo tipo de inversores.

Muchas de las ventajas de los partícipes de esta tipología de fondos de inversión son las mismas que adquirirían al invertir en fondos de inversión tradicionales: diversificación, sencillez operativa e inversión global (invertir en cualquier mercado del mundo sin tener conocimientos sobre las empresas que cotizan en ellos).

Pero cabe destacar importantes diferencias a favor del inversor en ETF: 
- Valoración en tiempo real. Los fondos cotizados tienen un precio o cotización en todo momento a lo largo de la sesión bursátil, a diferencia de lo que sucede con el resto de los fondos de inversión, cuyas participaciones son suscritas o reembolsadas al valor liquidativo diario que se obtiene cada día.

- Liquidez. La adquisición y venta de fondos cotizados tiene una liquidez inmediata, ya que pueden ser adquiridos o vendidos en cualquier momento durante la fase del mercado abierto del mercado continuo. Esto no es posible en los fondos de inversión no cotizados. Esta circunstancia se debe también a la actuación de los especialistas encargados de fomentar la liquidez de los ETF, como se comentará más adelante.

- Mayor transparencia. Durante la sesión bursátil se conoce en todo momento la composición de la cartera de valores de los ETF, así como su precio de cotización o valor liquidativo.

- Posible reparto de dividendos. En la mayoría de los fondos de inversión tradicionales de carácter financiero los rendimientos de los títulos que forman parte de la cartera se reinvierten. $A$ diferencia de ello, los fondos cotizados de renta variable pueden repartir dividendos, atendiendo al criterio del gestor del ETF.

- Gestión pasiva. A los inversores que consideran que los mercados son prácticamente imposibles de batir en el largo plazo (mercados eficientes) les permite adoptar una postura pasiva en la inversión y obtener la rentabilidad del mercado, representado por el índice bursátil replicado.

- Gestión activa. Con los ETF también es posible realizar operaciones de trading o de especulación a corto, medio o largo plazo, como si se tratara de acciones. Se pueden formar carteras de ETF, sobreponderando o infraponderando los diferentes países, mercados o sectores en función de su sobrevaloración o infravaloración.

- Estrategias sofisticadas. Además de las estrategias activa y pasiva, los fondos cotizados permiten una amplia gama de estrategias de especulación, e incluso de cobertura con instrumentos financieros derivados (opciones y futuros) sobre ETF.

- Menores costes. La adquisición de fondos cotizados se realiza al mismo coste que la compra o venta de acciones. No se les aplica las comisiones de suscripción y de reembolso que son habituales en los fondos de inversión no cotizados.

Además, la inversión en un ETF implica una única operación de compra que se invierte en una cesta de valores, frente al elevado coste que supondría para el inversor particular reproducir un índice bursátil comprando, en las mismas proporciones, el conjunto de valores que constituyen el índice y soportar elevadas comisiones. De esta manera, las economías de escala y de gestión alcanzadas por los fondos se traducen en una reducción de costes. No obstante, estos fondos soportan una serie de comisiones que se detallan a continuación:

- Comisión de intermediación o corretaje. Es la cobrada por el intermediario (sociedad o agencia de valores) al realizar la operación de compraventa. En el caso de que la orden se curse a través de una entidad que no sea miembro de mercado habrá que añadir un porcentaje adicional. Esta comisión es de libre fijación por parte del intermediario.

- Canon de Bolsa. Según los tipos establecidos por la Sociedad de Bolsas, variable en función del efectivo de la operación.

- Canon de Iberclear. Iberclear es el organismo de compensación y liquidación de valores. El canon por este concepto fluctúa en función del efectivo de la operación.

- Comisión de gestión. Es la aplicada por la gestora en función del patrimonio del fondo, de sus rendimientos, o de ambos.

- Comisión de administración, depósito o custodia de valores. Se aplica normalmente con periodicidad semestral y se cobra por la administración informática de las participaciones, la gestión de dividendos, la asistencia a juntas, el canje de valores...

Los agentes que intervienen en la operativa de los fondos de inversión cotizados, además de los $\triangleright$ 
partícipes (personas físicas, jurídicas u otras Instituciones de Inversión Colectiva que realizan aportaciones al fondo), son: el emisor y el gestor, el depositario y los especialistas.

El emisor y gestor es el que constituye el fondo cotizado y se encarga de la promoción o difusión, emisión y reembolso de las participaciones, toma de decisiones de inversión y desinversión, mantiene la cartera ajustada para replicar al índice de referencia, lleva la administración, gestión y representación de los fondos, así como el cálculo del valor liquidativo indicativo.

Por otro lado, existe una sociedad depositaria, (distinta de la sociedad gestora), que se encarga del depósito y custodia de los valores o activos adquiridos por el ETF, así como de la vigilancia y el control de la sociedad gestora.

Cabe destacar que en este mercado aparece la figura de los especialistas (market makers), entidades miembros del mercado encargadas de comprar o vender participaciones en el fondo a la sociedad gestora para cumplir su obligación de otorgar liquidez al producto. Los especialistas favorecen la difusión y el proceso de formación de precios, de manera que trabajan con una horquilla máxima de precios que ofrecen al inversor interesado en contratar, permitiendo la realización de negociaciones en el mercado secundario a precios en línea con el valor liquidativo indicativo del ETF.

Adicionalmente, en el mercado primario, facilitan que el número de participaciones disponibles de cada fondo en cada momento sea el adecuado, de manera que cada valor que se negocia en un segmento debe tener al menos un especialista. La actuación simultánea de los especialistas en los mercados primario y secundario permite que el precio se mantenga en niveles cercanos al valor patrimonial del fondo a través del arbitraje. Por lo tanto, los especialistas son creadores artificiales de liquidez.

Los ETF son supervisados por la CNMV (Comisión Nacional del Mercado de Valores), organismo público al que corresponde la vigilancia, supervisión e inspección de los mercados de capitales velando por su transparencia, por la correcta formación de precios y por la protección de los inversores. Además, con el objetivo de reforzar su seguridad, en 2001 se creó el Fondo de Garantía de Inversores (FOGAIN), que acumula las aportaciones realizadas por las sociedades adheridas, y cubre hasta un máximo de 100.000 euros de pérdida por inversor en caso de que una sociedad comercializadora entrara en concurso de acreedores, o la CNMV realizara una declaración de insolvencia.

\subsection{Normativa y requisitos para su admisión en el mercado bursátil}

Al tratarse de fondos de inversión, los ETF se encuentran regulados por la Ley 35/2003 de Instituciones de Inversión Colectiva y los Reales Decretos 1309/2005 y 1082/2012.

Los requisitos para la admisión a negociación en el mercado bursátil de las participaciones de este tipo de fondos (Real Decreto 1082/2012, artículo 79) son:

- Obtener la autorización de la CNMV.

- El objetivo de la política de inversión debe ser reproducir un índice financiero con una composición suficientemente diversificada, de fácil reproducción, de un mercado o conjunto de valores de referencia con una adecuada difusión.

- Los valores del fondo deben ser activos aptos para la inversión.

- La sociedad gestora determina la paridad, la composición y/o la cantidad de efectivo que tiene que ser intercambiada por participaciones.

- Deben existir entidades que asuman posiciones compradoras o vendedoras de participaciones con un diferencial máximo de precios (market makers) para facilitar que la cotización sea lo más próxima posible al valor liquidativo estimado.

- Se debe realizar una difusión adecuada del fondo a través de la sociedad rectora de la bolsa en la que cotice. 


\begin{tabular}{|c|c|}
\hline \multicolumn{2}{|r|}{$\begin{array}{l}\text { CUADRO } 1 \\
\text { TIPOLOGÍA DE ETF }\end{array}$} \\
\hline Tipología & Descripción \\
\hline Sobre índices de renta variable & Replican índices de las principales bolsas mundiales: IBEX-35, FTSE 100, CAC 40, Dow Jones... \\
\hline Sobre índices de renta fija & Replican índices de activos de renta fija (obligaciones y bonos gubernamentales y privados). \\
\hline Monetarios & $\begin{array}{l}\text { Indexados a activos de deuda estatal a corto plazo y otros activos del mercado interbancario de } \\
\text { máxima calificación crediticia. }\end{array}$ \\
\hline Sobre índices de materias primas & $\begin{array}{l}\text { Estos no aparecen hasta principios del siglo XXI (Corbet y Twomey, 2014) y actúan sobre los } \\
\text { mercados de materias primas. }\end{array}$ \\
\hline Sobre índices nacionales & $\begin{array}{l}\text { Indexados a mercados nacionales, ya sean de renta fija o variable. En el caso de España su } \\
\text { referencia es el IBEX-35. }\end{array}$ \\
\hline Sobre índices regionales & $\begin{array}{l}\text { Indexados a índices de renta fija o variable que representan empresas de determinadas regiones } \\
\text { dentro de un país, continente o grupo económico, como por ejemplo el Índice } \mathrm{MSCl} \text { del Pacífico. }\end{array}$ \\
\hline Sobre índices globales & $\begin{array}{l}\text { Indexados a índices de renta fija o variable a nivel mundial (Ejemplo: MSCI World Index, S\&P } \\
\text { Global } 100 \ldots) .\end{array}$ \\
\hline Sobre índices según su capitalización & $\begin{array}{l}\text { Representan grupos de valores en función del nivel de capitalización bursátil (alta, media o baja); } \\
\text { por ejemplo, IBEX Medium Cap o IBEX Small Cap en España. }\end{array}$ \\
\hline Sectoriales & $\begin{array}{l}\text { Replican índices de los diferentes sectores empresariales; por ejemplo, respecto al índice S\&P } \\
\text { Global Clean Energy Index. }\end{array}$ \\
\hline Apalancados & $\begin{array}{l}\text { Replican el índice con una determinada proporción de apalancamiento, incrementando las } \\
\text { ganancias o pérdidas según la proporción, pero asumiendo mayor riesgo. }\end{array}$ \\
\hline Inversos & Operan en sentido contrario al del mercado. \\
\hline Inversos y apalancados & Combinan las dos características anteriores. \\
\hline Alternativos & $\begin{array}{l}\text { Sobre volatilidad, inflación, índices que agrupan empresas susceptibles de sufrir transformaciones } \\
\text { (OPA, fusiones...); por ejemplo, sobre Credit Suisse Merger Arbitrage Liquid Index. }\end{array}$ \\
\hline Agrupados por estilo de inversión & $\begin{array}{l}\text { De crecimiento, de dividendos, agresivos, según horizonte temporal; por ejemplo, el estilo target } \\
\text { retirement date tiene el objetivo de obtener unos beneficios para afrontar la jubilación. }\end{array}$ \\
\hline ición & ación obtenida de BME (www.bolsasymercados.com). \\
\hline
\end{tabular}

\subsection{Tipología}

Los ETF se diferencian según el índice que replican o el estilo de inversión que adoptan. Una clasificación, en la que las categorías no son excluyentes entre sí, podría ser la que se incluye en el Cuadro 1.

\subsection{Fiscalidad}

Respecto a las personas físicas, la diferencia entre el importe obtenido en la venta y el satisfecho en la compra de un ETF se considera una ganancia o pérdida patrimonial, que se integra en la base imponible del ahorro o en la base general, dependiendo del plazo en el que se haya generado, del Impuesto sobre la Renta de las Personas Físicas. En caso de que varios titulares compartiesen la propiedad de las participaciones, los rendimientos se consideran obtenidos en proporción a la titularidad que cada uno tenga asignada. Si no se ha señalado expresamente ningún porcentaje, se presume titularidad a partes iguales.
Además, desde el 1 de enero de 2015 (Ley 26/2014) no existe distinción por antigüedad, y el tipo de gravamen aplicable es del 20 por 100 para los primeros 6.000 euros, el 22 por 100 para la ganancia adicional hasta los 50.000 euros, y el 24 por 100 para el importe que exceda los 50.000 euros. Esta retención será también aplicable a los dividendos repartidos, en caso de que existan, sin que haya lugar a la exención de los primeros 1.500 euros, tras la entrada en vigor de la normativa.

Por último, a diferencia de los fondos de inversión tradicionales, no se aplica retención a cuenta sobre las ganancias patrimoniales de su transmisión; sin embargo, no se les aplica la exención de tributación por el traspaso de participaciones, del que sí se benefician los fondos tradicionales.

\subsection{Ventajas y riesgos}

La inversión en ETF, al igual que en cualquier otro producto de inversión colectiva, supone una serie de ventajas y desventajas. Respecto a las $\square$ 
ventajas, en primer lugar, estos fondos se adecuan al perfil del inversor, de manera que los inversores pueden elegir un fondo u otro, según su vocación inversora; es decir, según el riesgo que se está dispuesto a asumir, el grado de diversificación deseado, el tipo de activo en el que invertir, el nivel de rentabilidad deseado, etcétera.

En segundo lugar, estos productos se caracterizan por un elevado nivel de información y transparencia. Cada fondo debe poseer un folleto informativo con las principales características, información sobre la evolución de su patrimonio y la composición de la cartera con periodicidad anual. Este folleto es proporcionado por las sociedades gestoras a los partícipes.

En tercer lugar, el inversor puede observar el precio y sus fluctuaciones en todo momento a lo largo de la sesión bursátil, al contrario que en el resto de fondos de inversión, cuyas participaciones son suscritas y reembolsadas al valor liquidativo diario que se obtiene al cierre. El único posible desajuste que se puede producir es en los ETF sobre índices de acciones, concretamente el día de reparto de dividendos, pero el desajuste se subsana una vez repartidos los dividendos. Respecto a estos últimos, a diferencia de la mayoría de fondos de inversión, que siguen una política de reinversión de dividendos, los ETF de renta variable pueden repartir dividendos si el gestor del ETF lo considera oportuno.

Por otro lado, los riesgos de una inversión de estas características son los mismos que en una inversión en activos del mercados secundario (acciones, bonos...). Por lo tanto, este producto está orientado a clientes que estén dispuestos a asumir los riesgos habituales de entrar en el mercado, de manera que se debe adecuar el perfil de riesgo del inversor con el perfil de riesgo del producto. Sin embargo, la diversificación de la cartera modelo hace que sea, por lo general, un producto de riesgo moderado.

En concreto, podemos diferenciar distintos riesgos en función de si el índice replicado es de renta fija o de renta variable. Respecto a los ETF sobre renta variable, el gestor se debe enfrentar principalmente a los dos tipos de riesgo en los que se incurre al contratar acciones: el riesgo específico y el riesgo sistemático. El riesgo específico dependerá de cada título y de las condiciones de la empresa que lo emita (sector de actividad, solvencia, endeudamiento, planes de crecimiento...), por lo que con una diversificación adecuada se puede reducir, e incluso eliminar. Por otro lado, el riesgo sistemático no es diversificable, ya que no depende de las características del título, sino de los factores generales del mercado, como la evolución económica o acontecimientos políticos, por lo que afectarán al conjunto total de la cartera.

Respecto a los ETF sobre índices de renta fija, los principales riesgos a tener en cuenta son: el riesgo de tipo de cambio, el riesgo país, el riesgo de crédito y, según el tipo de activo, el riesgo de liquidez.

Por último, en los ETF con un horizonte de inversión temporal corto, como los monetarios, se debe prestar atención al riesgo de reinversión, ya que al llegar el vencimiento del activo replicado, el gestor debe encontrar otro de similares características en un periodo de tiempo breve. No obstante, debido a la gran oferta existente de este tipo de activos en el mercado (REPO de deuda, letras a corto plazo, etcétera), este riesgo suele ser mínimo.

\section{Funcionamiento y réplica del índice}

Según el índice que se pretende replicar, el funcionamiento del fondo varía, ya que existen diversos métodos de réplica. Dado que la réplica de índices bursátiles es la más común, en este apartado nos centramos en las formas de replicar un índice bursátil. Existen diversos métodos para replicar un índice, siendo los más habituales: réplica física, réplica mediante optimización y réplica sintética.

La réplica física consiste en la compra de todos los valores que conforman el índice, o bien una parte representativa de ellos. Por tanto, se puede $\triangle$ 
distinguir entre réplica completa (full replication) y mediante muestreo estratificado (stratified sampling).

Respecto a la réplica completa, ésta consiste en adquirir todos los valores que constituyen el índice de acuerdo a su ponderación en el mismo. Es muy apropiada para replicar índices compuestos por pocos valores. Permite obtener el rendimiento del índice alejándose muy poco (bajo tracking error). Por otro lado, la réplica mediante muestreo estratificado (stratified sampling) consiste en componer una cesta de valores mediante un subconjunto de títulos constituyentes del índice. Los valores se agrupan en función de una serie de características, como sector de actividad, valor, expectativas de crecimiento, reparto de dividendos o capitalización. A continuación, a cada característica se le asigna una ponderación en función del peso que ese grupo de valores tiene en el índice. Finalmente, se selecciona un valor de cada sección de manera aleatoria y se pondera por el porcentaje que le corresponde según su sector. La réplica estratificada permite a la cartera que coincida con las características fundamentales del índice, sin realizar una réplica completa.

En segundo lugar, la réplica mediante optimización, también llamada sampled optimization, se basa en el uso de un programa-algoritmo de optimización que elabora carteras más eficientes que las determinadas mediante muestreo estratificado.

Por último, la réplica sintética no implica la compra física de los títulos del índice, por lo que se estructurará mediante activos sustitutivos; es decir, valores que no tienen por qué coincidir con los valores compuestos por el índice que se replica, pero que tienen un comportamiento y unas características muy similares. Asimismo, también pueden estructurarse mediante swap-based replication. Esta técnica consiste en un contrato de swap, o permuta financiera, que se lleva a cabo en mercados OTC (no regulados), en los que una entidad financiera se compromete a pagar a la gestora el rendimiento del índice al que esté referenciado el ETF, a cambio de las plusvalías que genere el colateral. Lo más usual es utilizar un swap de rendimiento entre dos índices; por ejemplo, recibir el rendimiento del índice Dow Jones a cambio de dar el rendimiento del IBEX-35.

La réplica sintética implica menores costes, menor tracking error, la eliminación del rebalanceo del índice y los problemas derivados por el pago de dividendos. La estructura planteada en la réplica sintética es, en términos de rentabilidad, más eficiente, pero conlleva unos mayores riesgos, ya que al tratarse de un contrato OTC, no tiene el respaldo de una posición directa larga en las compañías del índice, y su complejidad es mayor, por lo que puede dificultar el acceso a pequeños inversores. Por ello, la réplica más extendida es la réplica física.

Aclarar que los índices susceptibles de que su comportamiento diario sea replicado en un ETF son aquellos que pueden ser utilizados como subyacentes de un producto derivado (índices denominados de segunda generación) o inversos y apalancados (denominados de tercera generación). Los apalancados, tal y como indica Shum et al. (2015), son unos productos nuevos, ya que aparecieron en 2006. En el caso del mercado de valores español, existen los siguientes índices de tercera generación:

- IBEX 35 inverso: se compone de los mismos valores que el índice IBEX 35, y replica el comportamiento diario inverso del índice IBEX 35 con dividendos mediante un término inverso, e incorpora un elemento de inversión en renta fija libre de riesgo.

- IBEX 35 doble inverso.

- IBEX 35 inverso triple.

- IBEX 35 inverso por cinco.

- IBEX 35 doble apalancado: se compone de los mismos valores que el índice IBEX 35, y duplica el comportamiento diario del índice IBEX 35 tradicional, incorporando un elemento de financiación para alcanzar el nivel de apalancamiento necesario.

- IBEX 35 doble apalancado bruto: duplica el comportamiento diario del IBEX 35 con dividendos.

- IBEX 35 doble apalancado neto: replica y apalanca por dos el IBEX 35 con dividendos netos (descontando la retención). 
GRÁFICO 1

EVOLUCIÓN DEL VOLUMEN DE EFECTIVO NEGOCIADO Y DEL NÚMERO DE OPERACIONES EN LOS ETF ESPAÑOLES DE 2006 A 2014

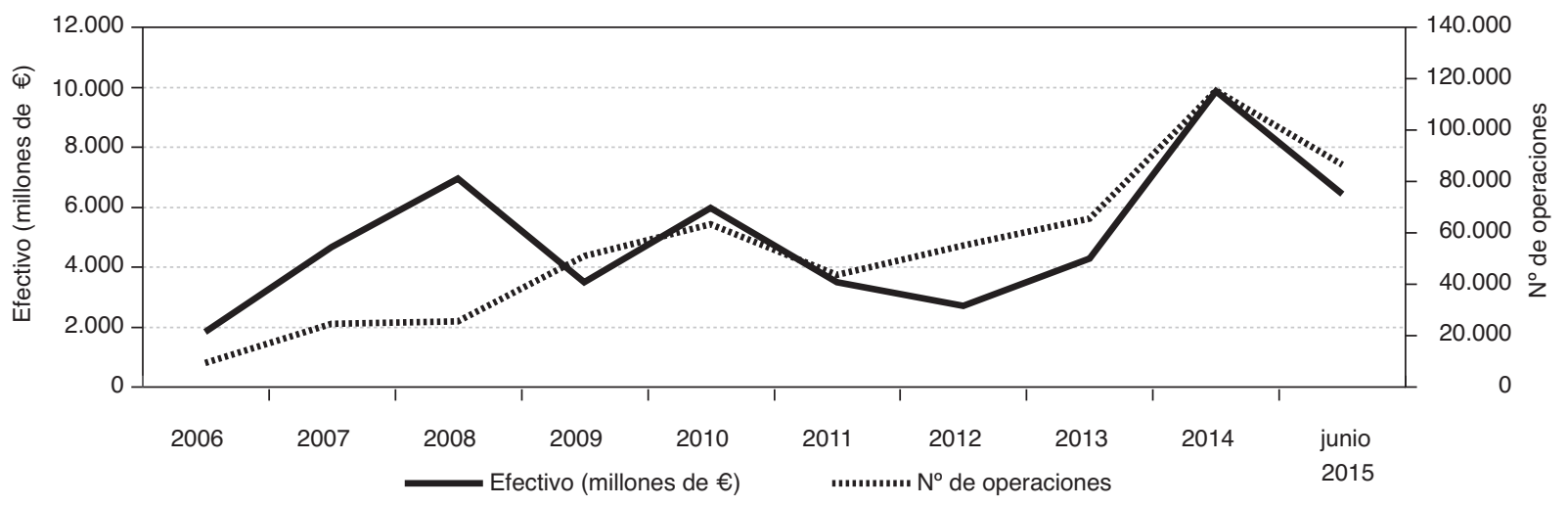

Fuente: elaboración propia a partir de datos de BME (www.bolsasymercados.es).

- IBEX 35 apalancado por tres.

- IBEX 35 apalancado neto por tres.

- IBEX 35 apalancado neto por cinco.

\section{Análisis del mercado español de los ETF}

\subsection{Cifras generales: negociación, número de fondos e inversión}

A pesar de que los ETF son un producto novedoso en el mercado bursátil español, estos han experimentado una evolución muy positiva a lo largo de los nueve años de funcionamiento que llevan operando en España, alcanzando una inversión superior a los 44.560 millones de euros en junio de 2015, utilizándose tanto para canalizar el ahorro, como para la especulación o la cobertura.

Desde sus comienzos en 2006, tal y como se observa en el Gráfico 1, los ETF tuvieron una notable acogida en el mercado, triplicando su volumen de negociación de 2006 a 2008, pasando de una negociación inferior a los 1.800 millones de euros a casi unos 7.000 millones de euros. No obstante, este producto también se ha visto afectado por la crisis financiera, disminuyendo el volumen de negociación desde 2008 a 2012, descendiendo hasta los 2.700 millones de euros, a pesar de que la recuperación bursátil favoreció un repunte en 2010. No obstante, durante los años 2013 y 2014

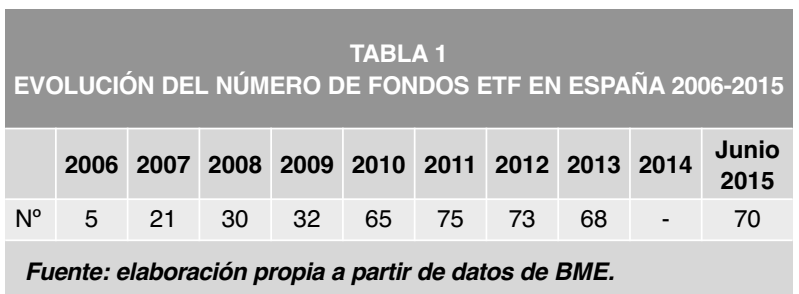

el producto ha experimentado una recuperación destacable, llegando a máximos históricos de negociación con un volumen de efectivo superior a los 9.800 millones de euros en 2014. De hecho, solo en la primera mitad de 2015 , la negociación ha superado los 6.400 millones de euros, y se observa una trayectoria creciente continua en el número de operaciones (a pesar del descenso en 2011), desde 9.100 operaciones en 2006, hasta más de 115.100 en 2014. Por tanto, se observa cómo este producto se está afianzando en el mercado y se está convirtiendo en una inversión alternativa cada vez más factible.

Respecto al número de fondos ETF existentes en España, la Tabla 1 muestra su evolución desde 2006 hasta junio de 2015. En ella podemos apreciar que en el año de su aparición, 2006, sólo existían 5 fondos, pero en 2007 la cifra se elevó hasta 21 fondos, valor que ha ido creciendo hasta alcanzar un máximo de 75 en 2011. A pesar de que el número de fondos ha ido disminuyendo desde entonces, en junio de 2015 esta cifra se elevaba a 70 ETF.

Considerando las cifras de inversión, el Gráfico 2 muestra la evolución del patrimonio invertido $\triangleright$ 


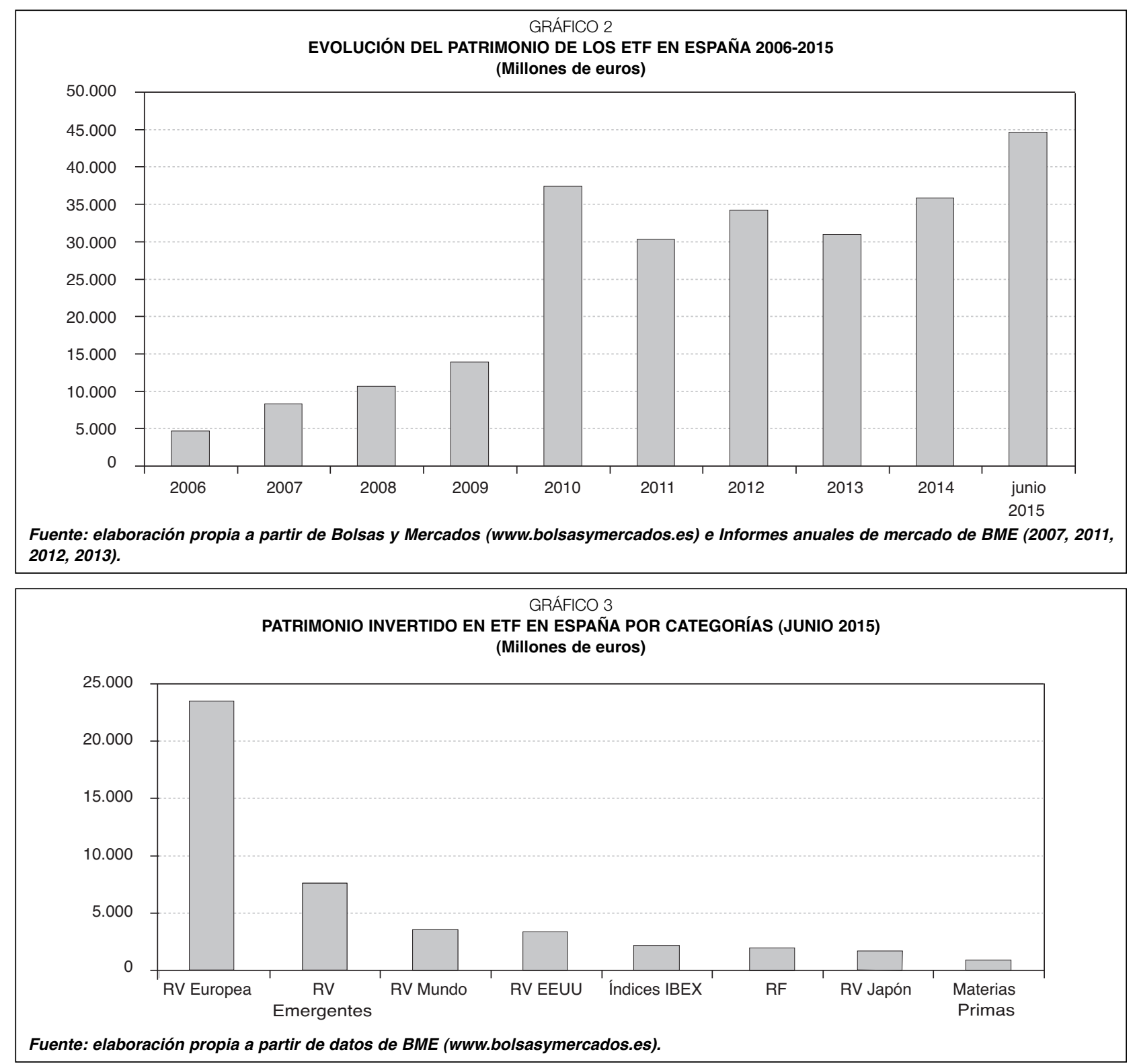

desde 2006 hasta junio de 2015. En dicho gráfico se aprecia una evolución constante positiva hasta 2010, destacando el gran crecimiento de 2009 a 2010, periodo en el que se pasó de una inversión superior a los 13.000 millones de euros en 2009 a más de 37.000 millones de euros en 2010. Sin embargo, en 2011 se hace patente la crisis, con un descenso de la inversión (30.200 millones de euros), volviendo a crecer en 2012 (34.100 millones de euros), y a pesar del nuevo descenso en 2013, existe un crecimiento muy positivo a lo largo de los últimos dos años, alcanzando máximos históricos de 44.593 millones de euros en junio de 2015.

\subsection{Inversión según categorías}

Los ETF en España se dividen en 8 categorías de inversión, según el tipo de índice que replican. Concretamente, las categorías existentes en la actualidad son: fondos que replican índices IBEX; fondos que replican índices de renta variable europea, de Estados Unidos y emergentes; fondos que replican índices de renta variable globales; fondos que replican índices de renta fija; fondos que replican índices de renta variable de Japón, y fondos que replican índices de materias primas.

La inversión entre las categorías es bastante dispar, tal y como muestra el Gráfico 3. 

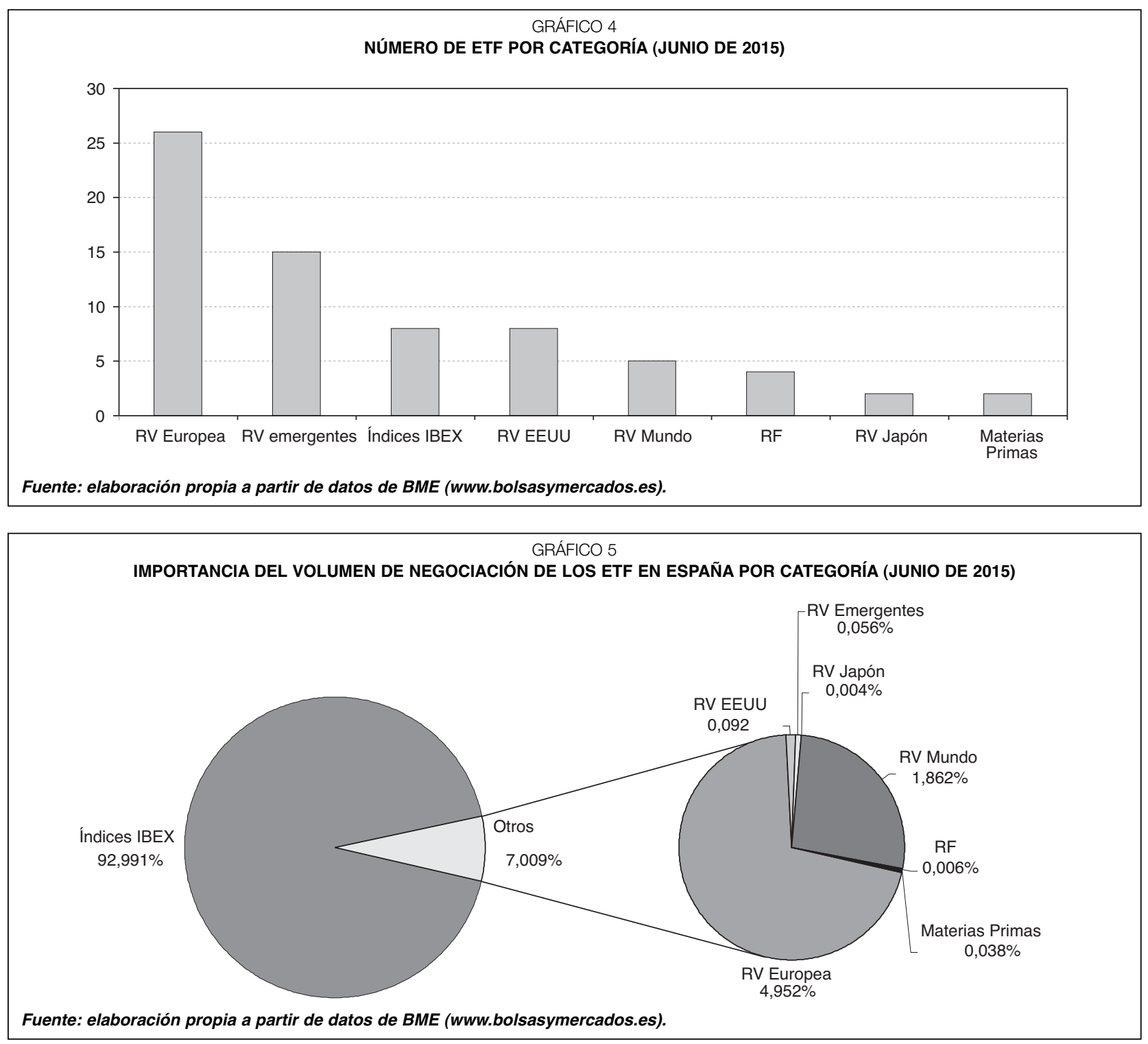

En el Gráfico 3 se observa que la categoría más importante a junio de 2015 es la de los fondos que replican índices de renta variable (RV) europeos (sin incluir a los que replican el IBEX), con una inversión total superior a los 23.400 millones de euros, y representando un 52,5 por 100 del mercado. En segundo lugar se encuentran los fondos que replican índices de renta variable de países emergentes con más de 7.500 millones de euros invertidos, representando el 17 por 100 del mercado. En tercer y cuarto lugar se posicionan los de índices estadounidenses y globales, con más de 3.500 y 3.400 millones de euros, respectivamente.

Respecto al mercado nacional, el patrimonio invertido en ETF que replican índices de la familia
IBEX supera los 2.130 millones de euros. Los de renta fija (RF), renta variable de Japón y materias primas presentan un peso residual, con una inversión superior a los $1.900,1.600$ y 877 millones de euros, respectivamente.

El Gráfico 4 recoge el número de fondos existentes por categoría en junio de 2015.

Comparando el patrimonio invertido (Gráfico 3) con el número de fondos por categoría (Gráfico 4), se observa coincidencia entre las primeras y últimas posiciones. En concreto, los fondos de índices de renta variable europea son los más numerosos, existiendo 26 ETF. A continuación se encuentran los fondos de índices de renta variable emergente, con 15 fondos. Sin embargo, en tercera y cuarta $D$ 
TABLA 2

PATRIMONIO INVERTIDO EN FONDOS ETF INDEXADOS A ÍNDICES IBEX (JUNIO 2014 Y 2015)

\begin{tabular}{|c|c|c|}
\hline \multirow{2}{*}{ Fondo } & \multicolumn{2}{|c|}{ Patrimonio (euros) } \\
\hline & $30 / 06 / 2014$ & $30 / 06 / 2015$ \\
\hline LYXOR UCITS ETF IBEX 35 (DR) & 893.080 .634 & 1.036 .851 .404 \\
\hline DB X-TRACKERS IBEX 35® UCITS ETF (DR) 1C & 671.066 .899 & 522.242 .213 \\
\hline ACCION IBEX 35 ETF, FI COTIZ, ARM. ......................... & 288.674 .524 & 390.438 .850 \\
\hline LYXOR UCITS ETF IBEX 35 DOBLE APALANCADO................... & 47.866 .490 & 94.610 .687 \\
\hline LYXOR UCITS ETF IBEX 35 DOBLE INV.DIARIO & 20.087 .633 & 23.519 .733 \\
\hline LYXOR UCITS ETF IBEX 35 INVERSO DIARIO & 18.137.558 & 20.064 .976 \\
\hline 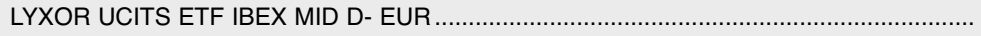 & 14.740 .964 & 12.034 .553 \\
\hline 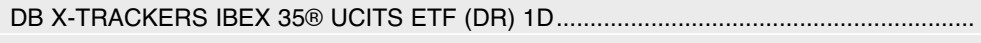 & - & 30.434 .433 \\
\hline Total & 1.953.654.703 & 2.130.196.848 \\
\hline
\end{tabular}

\begin{tabular}{|c|c|}
\hline \multicolumn{2}{|c|}{$\begin{array}{c}\text { TABLA } 3 \\
\text { EFECTIVO NEGOCIADO EN LOS ETF CON SUBYACENTE IBEX (JUNIO 2015) }\end{array}$} \\
\hline Fondo & Efectivo (euros) \\
\hline 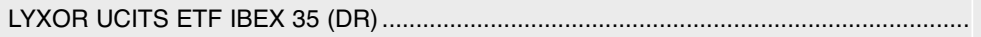 & 387.035 .274 \\
\hline LYXOR UCITS ETF IBEX 35 DOBLE APALANCADO & 219.659 .402 \\
\hline DB X-TRACKERS IBEX 35® UCITS ETF (DR) 1C & 118.817 .480 \\
\hline ACCION IBEX 35 ETF, FI COTIZ, ARM. ................................. & 113.869 .945 \\
\hline LYXOR UCITS ETF IBEX 35 DOBLE INV.DIARIO & 104.685 .985 \\
\hline LYXOR UCITS ETF IBEX 35 INVERSO DIARIO & 23.139 .470 \\
\hline DB X-TRACKERS IBEX 35® UCITS ETF (DR) 1D & 18.017.169 \\
\hline LYXOR UCITS ETF IBEX MID D- EUR & 4.441 .895 \\
\hline Total. & 989.666 .620 \\
\hline
\end{tabular}

posición se encuentran los fondos de índices IBEX y de renta variable estadounidense, ambos con 8 fondos. En las cuatro últimas posiciones, existen 5 fondos que invierten en índices globales, 4 en índices de renta fija, 2 en índices de renta variable de Japón y 2 en índices de materias primas. Estas cifras evidencian una notable concentración en el mercado español de ETF, ya que sólo 71 fondos gestionan más de 44.500 millones de euros.

No obstante, si comparamos el efectivo negociado según categorías (Gráfico 5), observamos que más del 92 por 100 del volumen de negociación corresponde a los fondos de índices IBEX, siendo la negociación del resto mucho menor. Por tanto, a pesar de que la categoría IBEX muestra un menor patrimonio y número de fondos, podemos concluir que estos fondos son comercializados de manera más frecuente, de manera que el resto de categorías estarían más orientadas a la inversión a medio y largo plazo, con una rotación inferior.

\subsection{Los ETF de índices IBEX}

Dado que la mayor parte de la contratación se concentra en ETF de índices IBEX, en este apartado realizamos un análisis más detallado de esta tipología de fondos.

En primer lugar estudiamos el patrimonio gestionado por los 8 fondos existentes en esta categoría. La Tabla 2 recoge su inversión en junio de 2014 y de 2015.

En ella también se observa una evolución positiva a lo largo del último año, excepto en dos fondos (DB X-TRACKERS IBEX 1C y Lyxor UCITS ETF IBEX MID D-EUR). Por otro lado, destaca el fondo Lyxor UCITS ETF IBEX 35, con más de 1.036 millones de euros invertidos en junio de 2015, lo que supone una cuota de mercado, en esta categoría, del 48,7 por 100 . Este fondo es comercializado por Lyxor y está basado en la réplica física. Los fondos situados en segunda y tercera posición están gestionados por Deutsche Bank y BBVA, también se $\triangle$ 
basan en la réplica física, y poseen un 24 y 18 por 100 de la inversión (más de 522 y 390 millones de euros, respectivamente, en junio de 2015). Destacar que en cuarto lugar se encuentra el ETF sobre IBEX doble apalancado, también de Lyxor, que, debido a su perfil más propenso al riesgo, solo supone un 4 por 100 de la inversión. Asimismo, los ETF sobre IBEX inverso gozan de menos popularidad, representando menos del 1 por 100 de la inversión en esta categoría.

Respecto al volumen de efectivo negociado (Tabla 3), la importancia coincide con el patrimonio gestionado, siendo el fondo Lyxor UCITS ETF IBEX 35 el que presenta un mayor volumen de negociación.

\section{Conclusiones}

A pesar de la reciente aparición en España de los fondos de inversión cotizados en 2006, su inversión ha alcanzado una posición notable en el mercado español, superando los 44.560 millones de euros en apenas 9 años.

Los ETF son productos híbridos entre los fondos de inversión y los títulos de renta variable que replican a un índice financiero y cotizan en mercados regulados. Por tanto, se rigen por las mismas directrices que los fondos de inversión, pero su operativa se asemeja a la de las acciones, de manera que sus operaciones están dotadas de gran dinamismo y liquidez.

La gran ventaja de los ETF es que permiten la toma de posiciones de manera fácil y accesible en una amplia variedad de mercados, sectores, tipos de activos o zonas geográficas. La compra de un ETF supone tomar una exposición de manera directa en la tendencia del índice que se replica y, en consecuencia, en todos los valores que éste incluye. La facilidad de contratación (los inversores solo necesitan hacerse partícipes del fondo con una inversión mínima de una participación), junto con las ventajas de la operativa de los fondos de inversión, han hecho posible reunir en un mismo producto tanto a pequeños inversores como a inversores institucionales, consiguiendo ambos la misma rentabilidad.

Aunque estos productos no gozan de algunas de las ventajas fiscales que presentan los fondos de inversión, como el traspaso libre de impuestos de un fondo a otro, se caracterizan por una gran liquidez e inmediatez, ya que se conoce su cotización a tiempo real, y es posible invertir y desinvertir en cortos periodos de tiempo, a diferencia de otros productos. Además, al ser un producto ligado al mercado, los inversores saben que su inversión seguirá la misma evolución, o muy parecida, que el mercado que se está replicando.

Los ETF son un producto en expansión que ha experimentado un gran crecimiento a lo largo de los últimos años y que presenta atractivas diferencias con respecto a la inversión en Instituciones de Inversión Colectiva tradicional. Sus posibilidades de inversión son muy amplias, ya que no sólo puede usarse con objeto de rentabilizar puntas de tesorería, para pequeños o largos periodos de tiempo, sino también para operaciones de arbitraje o cobertura.

Entre las diferentes categorías existentes en España, los ETF con índices de renta variable europea como subyacente son los de mayor importancia, tanto a nivel de patrimonio gestionado (más de 23.400 millones de euros), como en número de fondos (26). No obstante, los que presentan un mayor volumen de negociación en el mercado español son los que replican índices IBEX, corroborando las buenas expectativas del mercado bursátil español, y mostrando el elevado conocimiento que los inversores tienen sobre el mercado doméstico.

Este trabajo analiza un producto de inversión alternativo que goza de respaldo legal y protección del inversor y da a conocer su funcionamiento, principales características y ventajas. Muestra que es un producto accesible a la totalidad de los inversores, de fácil comprensión, con unas rentabilidades atractivas y que todavía no ha tocado techo en cuanto a sus posibilidades de expansión y desarrollo.

Por tanto, los ETF son un producto atractivo para invertir en periodos como el actual, en los que $D$ 
inversiones tradicionales, como los depósitos bancarios o la renta fija pública, ofrecen escasa rentabilidad. De hecho, se prevé un aumento tanto de la demanda como de la oferta de ETF, ya que existen múltiples posibilidades de réplica de distintos índices, tanto de renta variable como de renta fija, sectoriales, según zonas geográficas, según capitalización, de materias primas, etcétera. No obstante, se debe tener muy presente el perfil de inversión del inversor, que debe ser capaz de asumir los riesgos derivados de las fluctuaciones de las cotizaciones en los mercados secundarios, sean de renta variable o de renta fija.

\section{Bibliografía}

[1] BLACKROCK (2014). ETP Landscape, September.

[2] $\operatorname{BME}(2007,2011,2012$ y 2013). Informe de mercado. Disponible en: www.bolsasymercados.es

[3] CORBET, S. y TWOMEY, C. (2014). «Have Exchange Traded Funds Influenced Commodity Market Volatility?». International Journal of Economics and Financial Issues, vol. 4, n² 2, pp. 323-335.
[4] ESPAÑA. LEY 35/2003, de 4 de noviembre, de Instituciones de Inversión Colectiva.

[5] ESPAÑA. LEY 26/2014, de 27 de noviembre, por la que se modifican la Ley $35 / 2006$, de 28 de noviembre, del Impuesto sobre la Renta de las Personas Físicas.

[6] ESPAÑA. REAL DECRETO 1309/2005, de 4 de noviembre, por el que se aprueba el Reglamento de la Ley 35/2003, de 4 de noviembre, de instituciones de inversión colectiva.

[7] ESPAÑA. REAL DECRETO 1082/2012, de 13 de julio, por el que se aprueba el Reglamento de desarrollo de la Ley 35/2003, de 4 de noviembre, de instituciones de inversión colectiva.

[8] GASTINEAU, G.L. (2010). The Exchange-Traded Funds Manual. John Wiley \& Sons.

[9] HURLIN, C.; ISELI, G.; PERIGNON, C. y YEUNG, S. (2015). "The Counterparty Risk Exposure of ETF Investors». HEC Paris Research Paper $n^{\circ}$ FIN2014-1050.

[10] SHUM, P.; HEJAZI, W.; HARYANTO, E. y RODIER, A. (2015). «Intraday Share Price Volatility and Leveraged ETF Rebalancing». Working paper. Recuperado el 22 de septiembre de 2015:

http://papers.ssrn.com/sol3/papers.cfm?abstract_ $i d=2161057$ 
